\title{
Subleading corrections to parity-violating pion photoproduction
}

\author{
Shi-Lin Zhu, ${ }^{1,2}$ S. Puglia, ${ }^{1,2}$ B. R. Holstein, ${ }^{3}$ and M. J. Ramsey-Musolf ${ }^{1,2,4}$ \\ ${ }^{1}$ Kellogg Radiation Laboratory 106-38, California Institute of Technology, Pasadena, California 91125 \\ ${ }^{2}$ Department of Physics, University of Connecticut, Storrs, Connecticut 06269 \\ ${ }^{3}$ Department of Physics, University of Massachusetts, Amherst, Massachusetts 01003 \\ ${ }^{4}$ Theory Group, Thomas Jefferson National Accelerator Facility, Newport News, Virginia 23606
}

(Received 22 March 2001; published 2 August 2001)

\begin{abstract}
We compute the photon asymmetry $B_{\gamma}$ for near threshold parity-violating (PV) pion photoproduction through subleading order. We show that subleading contributions involve a new combination of PV couplings not included in previous analyses of hadronic PV. We argue that existing constraints on the leading order contribution to $B_{\gamma}$-obtained from the PV $\gamma$-decay of ${ }^{18} \mathrm{~F}$ - suggest that the impact of the subleading contributions may be more significant than expected from naturalness arguments.
\end{abstract}

DOI: $10.1103 /$ PhysRevC.64.035502

\section{INTRODUCTION}

The parity-violating (PV) $\pi N N$ Yukawa coupling constant $h_{\pi}^{\text {eff }}$ is a key ingredient to the understanding of the PV nuclear interaction [1-5] (historically, this constant has been denoted as $f_{\pi}$ in the literature). A number of hadronic PV experiments have sought to determine the value of $h_{\pi}^{\text {eff }}[2,5-$ 9]. A particularly significant result has been obtained from measurements of photon polarization $P_{\gamma}$ in the PV $\gamma$ decay of ${ }^{18} \mathrm{~F}$ :

$$
h_{\pi}^{\mathrm{eff}}=(0.73 \pm 2.3) g_{\pi},
$$

where $g_{\pi}=3.8 \times 10^{-8}$ gives the scale of $g_{\pi}$ in the absence of weak neutral currents [1]. An explicit SU(6)/quark model analysis [1], as well as "naturalness" arguments (see below), would suggest that $h_{\pi}^{\text {eff }}$ should be closer to $10 g_{\pi}$. The results of the ${ }^{18} \mathrm{~F}$ measurement, which has been repeated by five different groups, is therefore surprising. The nature of the $h_{\pi}^{\text {eff }}$ puzzle is further complicated by two additional observations.

(i) The governing PV mixing matrix element in ${ }^{18} \mathrm{~F}$ can be related by isospin symmetry to two body component of the experimental rate for the analog $\beta$ decay ${ }^{18} \mathrm{Ne} \rightarrow{ }^{18} \mathrm{~F}+e^{+}$ $+\nu_{e}[10,12]$. Since $P_{\gamma}\left({ }^{18} \mathrm{~F}\right)$ is dominated by its sensitivity to $h_{\pi}^{\text {eff }}$, the bounds in Eq. (1) appear to be robust from the standpoint of many-body nuclear theory [2].

(ii) A measurement by the Boulder group of the nuclear spin-dependent PV effects in $6 \mathrm{~S}-7 \mathrm{~S}$ transitions in the ${ }^{133} \mathrm{Cs}$ atom has been used in order to extract a value for the cesium nuclear anapole moment (AM) [8]. Recently, a full two-body calculation of the cesium AM has been used to extract constraints on the long- and short-range components of the PV $N N$ interaction [11]. When combined with the constraints on the short-range PV $N N$ interaction, the cesium results imply a central value for $h_{\pi}^{\text {eff }}$ of $\sim 10 g_{\pi}$, in agreement with the "naturalness" estimate.

The status of $h_{\pi}^{\text {eff }}$ may be clarified by a slate of new experiments-suggested, planned, or currently underway: $\vec{n} p \rightarrow d \gamma$ at LANSCE [13], $\gamma^{*}, \gamma d \rightarrow n p$ at Jefferson Lab [14], the rotation of polarized neutrons in helium at NIST [9] as well as polarized Compton scattering processes $[15,16]$.
PACS number(s): 25.20.Lj, 11.30.Er, 11.30.Rd, 13.60.Le

Since these processes involve one- and few-body systems, one anticipates new constraints on the PV $N N$ interaction free from many-body uncertainties related to complex nuclei such as cesium or fluorine.

If the new experiments were to confirm the present ${ }^{18} \mathrm{~F}$ constraints on $h_{\pi}^{\text {eff }}$, then one should attempt to understand the nucleon structure dynamics responsible for the reduction from its "natural" size. At the same time, it would become necessary to account for the subleading chiral structure of the PV $\pi N N$ Yukawa interaction and its related observables. To that end, we recently computed the subleading chiral contributions to $h_{\pi}^{\text {eff }}$ [17]. At leading order, $h_{\pi}^{\text {eff }}$ is identical to the low-energy constant (LEC) $h_{\pi}^{1}$ appearing in the PV pionnucleon chiral Lagrangian [4]. The subleading contributions, which vanish in the chiral limit, involve a host of new LEC's whose effect on $h_{\pi}^{\text {eff }}$ is fortuitously enhanced. A similar set of LEC's appear in anapole moment contributions to the radiative corrections to backward angle PV ep scattering. These corrections, which have recently been determined by the SAMPLE Collaboration [19], appear to be considerably larger than one's theoretical expectation [18]. Thus, there appear to be several hints that the chiral expansion for hadronic PV may not behave as one naively expects.

With this situation in mind, we consider in this article the subleading chiral contributions to another PV observable: the polarization asymmetry $B_{\gamma}$ for the charged pion photoproduction process

$$
\vec{\gamma}\left(q^{\mu} ; \epsilon^{\mu}\right)+p\left(P_{i}^{\mu}\right) \rightarrow \pi^{+}\left(k^{\mu}\right)+n\left(P_{f}^{\mu}\right),
$$

which will be the focus of the proposed JLab study. Here, $q^{\mu}=(\omega, \mathbf{q}), P_{i}^{\mu}, k^{\mu}=\left(\omega_{\pi}, \mathbf{k}\right)$, and $P_{f}^{\mu}$ are the center-ofmass four-momenta of photon, proton, pion and neutron, respectively, and $\epsilon^{\mu}$ is the photon polarization vector. The asymmetry $B_{\gamma}$, which arises from the interference of PV and parity conserving (PC) amplitudes, was first studied in the context of the conventional meson-exchange framework for hadronic PV in Refs. [23,24]. Recently, Chen and Ji (CJ) proposed a measurement of $B_{\gamma}$ at the Jefferson Lab and recast the earlier analyses in the context of heavy baryon chiral perturbation theory (HBCPT) [21,22]. The authors empha- 
sized that PV $\pi$ photoproduction accesses the PV $N N \pi$ interaction directly, whereas in nuclear observables it is contained within the PV NN potential. For the threshold region, where all external momenta are well below the chiral symmetry breaking scale $\Lambda_{\chi}=4 \pi F_{\pi} \sim 1 \mathrm{GeV}$, CJ obtain the "low-energy theorem" for the asymmetry

$$
B_{\gamma}\left(\omega_{\text {th }}, \theta\right)=\frac{\sqrt{2} F_{\pi}\left(\mu_{p}-\mu_{n}\right)}{g_{A} m_{N}} h_{\pi}^{1}
$$

and the corrections from terms higher order in the chiral expansion were estimated to be around 20\% [20]. The expression in Eq. (3) is consistent with the $h_{\pi}^{\text {eff }}$ dominance of $B_{\gamma}$ found in Ref. [24]. CJ also explored the kinematic behavior of $B_{\gamma}$, indicating that it could be large enough to be observed in a polarized photon beam experiment at Jefferson Laboratory.

In this paper, we show that inclusion of subleading contributions to the PV photoproduction amplitude leads to a chirally corrected low-energy theorem:

$$
B_{\gamma}\left(\omega_{\mathrm{th}}, \theta\right)=\frac{\sqrt{2} F_{\pi}}{g_{A} m_{N}}\left[\mu_{p}-\mu_{n}\left(1+\frac{m_{\pi}}{m_{N}}\right)\right] h_{\pi}^{1}-\frac{4 \sqrt{2} m_{\pi}}{g_{A} \Lambda_{\chi}} \bar{C},
$$

where the $m_{\pi} / m_{N}$ represents the first recoil corrections to the leading order PV and PC photoproduction amplitudes and $\bar{C}$ is a new PV LEC defined below. In terms of chiral counting, the result of $\mathrm{CJ}$ appears at $O\left(p^{0}\right)$ while the corrections arising in Eq. (4) occur at $O(p)$. We note that the recoil and $\bar{C}$ terms shown explicitly in Eq. (4) constitute the complete set of subleading contributions to the PV photoproduction amplitude, since the effects of loops as well as pole diagrams involving decuplet intermediate states arise at $O\left(p^{2}\right)$ and beyond.

At face value, the expression in Eq. (4) indicates that $B_{\gamma}$ is governed by two, rather than one, PV LEC's $-h_{\pi}^{1}$ and $\bar{C}$, with associated kinematic factors of nearly equal magnitude. The actual situation, however, is more subtle. The naturalness arguments which imply $h_{\pi}^{1}$ should be $\sim 10 g_{\pi}$ also lead one to expect $\bar{C} \sim g_{\pi}$. Thus, if these two LEC's were to have their natural size, the subleading contributions to $B_{\gamma}$ would generate the anticipated $10 \%$ effect. ${ }^{1}$ The results of the ${ }^{18} \mathrm{~F}$ experiment, on the other hand, imply that $h_{\pi}^{1}$ is strongly suppressed from its natural scale. In this case, one would expect $h_{\pi}^{1}$ and $\bar{C}$ to be of comparable importance. Given the present lack of a first principle QCD calculation of these two LEC's, it is up to experiment to settle the question. As noted in CJ, if $h_{\pi}^{1}$ were to have its natural size, then a $20 \%$ determination of $B_{\gamma}$ may be feasible at Jefferson Lab. On the other hand, a null result at this precision would be consistent with the ${ }^{18} \mathrm{~F}$ experiment and would imply the need of additional measurements to separate $h_{\pi}^{1}$ and $\bar{C}$.

\footnotetext{
${ }^{1}$ We thank the authors of CJ as well as J.L. Friar for clarification of this point.
}

In the remainder of this paper, we discuss the calculations leading to our conclusions. In Sec. II, we summarize the formalism for treating hadronic PV in HBCPT. Section III gives the calculation of the subleading contributions to the PV photoproduction amplitude. In Sec. IV, we discuss a field redefinition, first suggested in Ref. [29], which expresses the results of Sec. III in a compact manner. In Sec. V, we consider the expected magnitudes of the PV LEC's, relate these estimates to the earlier work of Ref. [24], and summarize our conclusions.

\section{HADRONIC PARITY VIOLATION IN CHIRAL PERTURBATION THEORY}

Before considering the heavy baryon expansion, it is useful to review the relevant PC and PV Lagrangians in the fully relativistic theory. For simplicity, we consider only $\pi, N$, and $\gamma$ interactions. In this case, for PC interactions one has

$$
\begin{aligned}
\mathcal{L}^{P C}= & \frac{1}{4} F_{\pi}^{2} \operatorname{Tr} D^{\mu} \Sigma D_{\mu} \Sigma^{\dagger}+\bar{N}\left(i \mathcal{D}_{\mu} \gamma^{\mu}-m_{N}\right) N \\
& +g_{A} \bar{N} A_{\mu} \gamma^{\mu} \gamma_{5} N+\frac{e}{\Lambda_{\chi}} \bar{N}\left(c_{s}+c_{v} \tau_{3}\right) \sigma^{\mu \nu} N F_{\mu \nu}^{+}+\cdots,
\end{aligned}
$$

where $\mathcal{D}_{\mu}$ is the chiral and electromagnetic (EM) covariant derivative, $\Sigma=\exp \left(i \vec{\tau} \cdot \vec{\pi} / F_{\pi}\right)=\xi^{2}, N$ is the nucleon isodoublet field and

$$
\begin{gathered}
A_{\mu}=\frac{i}{2}\left(\xi^{\dagger} \partial_{\mu} \xi-\xi \partial_{\mu} \xi^{\dagger}\right), \\
F_{\mu \nu}^{ \pm}=\frac{1}{2} F_{\mu \nu}\left(\xi \Lambda_{p} \xi^{\dagger} \pm \xi^{\dagger} \Lambda_{p} \xi\right), \\
\Lambda_{p}=\frac{1}{2}\left(1+\tau_{3}\right) .
\end{gathered}
$$

The relevant PV Lagrangians are $[4,18]$

$$
\begin{aligned}
\mathcal{L}^{P V}= & h_{V}^{0} \bar{N} A_{\mu} \gamma^{\mu} N+\frac{h_{V}^{1}}{2} \bar{N} \gamma^{\mu} N \operatorname{Tr}\left(A_{\mu} X_{+}^{3}\right) \\
& -\frac{h_{A}^{1}}{2} \bar{N} \gamma^{\mu} \gamma_{5} N \operatorname{Tr}\left(A_{\mu} X_{-}^{3}\right)-\frac{h_{\pi}^{1}}{2 \sqrt{2}} F_{\pi} \bar{N} X_{-}^{3} N \\
& +h_{V}^{2} \mathcal{I}^{a b} \bar{N}\left[X_{R}^{a} A_{\mu} X_{R}^{b}+X_{L}^{a} A_{\mu} X_{L}^{b}\right] \gamma^{\mu} N \\
& -\frac{h_{A}^{2}}{2} \mathcal{I}^{a b} \bar{N}\left[X_{R}^{a} A_{\mu} X_{R}^{b}-X_{L}^{a} A_{\mu} X_{L}^{b}\right] \gamma^{\mu} \gamma_{5} N \\
& +\frac{c_{1}}{\Lambda_{\chi}} \bar{N} \sigma^{\mu \nu}\left[F_{\mu \nu}^{+}, X_{-}^{3}\right]_{+} N+\frac{c_{2}}{\Lambda_{\chi}} \bar{N} \sigma^{\mu \nu} F_{\mu \nu}^{-} N \\
& +\frac{c_{3}}{\Lambda_{\chi}} \bar{N} \sigma^{\mu \nu}\left[F_{\mu \nu}^{-}, X_{+}^{3}\right]_{+} N,
\end{aligned}
$$


where

$$
\begin{gathered}
X_{L}^{a}=\xi^{\dagger} \tau^{a} \xi, \\
X_{R}^{a}=\xi \tau^{a} \xi^{\dagger}, \\
X_{ \pm}^{a}=X_{L}^{a} \pm X_{R}^{a},
\end{gathered}
$$

and where we follow the sign convention of Refs. [18,17]. The corresponding PC and PV Lagrangians involving $\Delta$ fields are given in Ref. [18].

Of the PV LEC's appearing in Eqs. (9), $h_{\pi}^{1}$ is the most familiar and has received the most extensive theoretical scrutiny $[1-5]$. In the context of chiral perturbation theory, the radiative corrections to $h_{\pi}^{1}$ were discussed extensively in [17], where it was pointed out that what nuclear PV experiments measure is an effective coupling $h_{\pi}^{\text {eff }}$ [17], which is a linear combination of LECs $h_{\pi}^{1}, h_{\Delta}, h_{A}^{(i)}$, etc. The commonly used "best value" $-\left|h_{\pi}^{1}\right|=5 \times 10^{-7}$-quoted in [1] corresponds to a large extent to a simple tree-level estimate without loop corrections. Estimates for $h_{V}^{i}$ and $h_{A}^{i}$ have been discussed in Refs. [4,17], though no analysis similar to that of [1] has been performed. To date, there have appeared no estimates of the PV $N N \pi \gamma$ constants $c_{i}$. Nevertheless, one expects the magnitude of these LEC's to be roughly a few times $g_{\pi}$.

For purposes of computing $B_{\gamma}$, it is necessary to expand the nonlinear Lagrangians of Eqs. (5) and (9) through one $\pi$ and one $\gamma$ order. The results for the PC interactions are familiar and we do not list them here. For the PV Lagrangians, we also include the leading ( $2 \pi)$ terms proportional to $h_{A}^{i}$ :

$$
\begin{aligned}
\mathcal{L}^{P V}= & -i h_{\pi}^{1} \pi^{+} p^{\dagger} n-\frac{h_{V}}{\sqrt{2} F} \bar{p} \gamma^{\mu} n D_{\mu} \pi^{+} \\
& +i \frac{h_{A}^{(1)}+\bar{h}_{A}^{(2)}}{F_{\pi}^{2}} \bar{p} \gamma^{\mu} \gamma_{5} p \pi^{+} D_{\mu} \pi^{-} \\
& +i \frac{h_{A}^{(1)}-\bar{h}_{A}^{(2)}}{F_{\pi}^{2}} \bar{n} \gamma^{\mu} \gamma_{5} n \pi^{+} D_{\mu} \pi^{-} \\
& -i e \frac{C}{\Lambda_{\chi} F_{\pi}} \bar{p} \sigma^{\mu \nu} F_{\mu \nu} n \pi^{+}+\text {H.c. }
\end{aligned}
$$

where

$$
\begin{gathered}
h_{V}=h_{V}^{0}+\frac{4}{3} h_{V}^{2}, \\
C=-2 \sqrt{2} c_{1}+\frac{1}{\sqrt{2}} c_{2} .
\end{gathered}
$$

Note that the LEC $h_{V}^{1}$ does not contribute to $\mathcal{L}^{P V}$ at this order. As noted in Ref. [29] and discussed in detail below, the effects of the $h_{V}^{i}$ Lagrangians on processes involving up to two pions and one photon can be absorbed into effective $C$ and $h_{A}^{i}$ type Lagrangians through $2 \pi$ order via an appropri- ate nucleon field redefinition. The reason is that when one integrates by parts the action corresponding to the $h_{V}$ term in Eq. (13), the integrand vanishes by the nucleon equations of motion. At $3 \pi$ order and beyond, however, the effects of the $h_{V}^{i}$ terms in Eq. (9) cannot be absorbed into other effective interactions via field redefinition. Thus, in the context of the complete nonlinear PV Lagrangian, the $h_{V}^{i}$ remain distinct LEC's. Consequently, we keep the $h_{V}$ dependence explicit in what follows.

\section{THE SUBLEADING CORRECTION TO THE ASYMMETRY}

In order to maintain proper chiral counting, we use the heavy baryon expansion of Eqs. (5) and (9). The motivation behind the use of heavy baryon chiral perturbation theory (HBCPT) is explained in detail in [20], and we follow the notations of this reference. Since we work in the nearthreshold region, we use the so-called "small-scale" expansion [25], i.e., we treat $\omega, \omega_{\pi},|k|, m_{\pi}, \delta=m_{\Delta}-m_{N}$, etc., as small quantities and characterize amplitudes by the number of powers of these terms, e.g., we count the term $\omega_{\pi} / q \cdot k$ as being $O\left(p^{-1}\right)$. The photon asymmetry arises from the interference of the parity conserving (PC) and PV amplitudes. In Ref. [20] the asymmetry was truncated at leading order, i.e., $O\left(p^{0}\right)$. In the present work we include the $O(p)$ correction, which arises dominantly from the PV vector $\pi N N$ couplings. As we show below, chiral loops contribute to the asymmetry only at $O\left(p^{2}\right)$ and higher. Hence, our truncation of the chiral expansion of the asymmetry is consistent and complete up to terms of $O(p)$.

The PC amplitudes which describe the charged photoproduction reaction are defined via

$$
\begin{aligned}
T^{P C}= & N^{\dagger}\left[i \mathcal{A}_{1} \vec{\sigma} \cdot \hat{\epsilon}+i \mathcal{A}_{2} \vec{\sigma} \cdot \hat{\mathbf{q}} \hat{\boldsymbol{\epsilon}} \cdot \hat{\mathbf{k}}\right. \\
& \left.+i \mathcal{A}_{3} \vec{\sigma} \cdot \hat{\mathbf{k}} \hat{\boldsymbol{\epsilon}} \cdot \hat{\mathbf{k}}+\mathcal{A}_{4} \hat{\boldsymbol{\epsilon}} \cdot \hat{\mathbf{q}} \times \hat{\mathbf{k}}\right] N,
\end{aligned}
$$

where $N$ is the proton Pauli spinor, $\vec{\sigma}$ are the Pauli spin matrices, and $\hat{\mathbf{q}}$ and $\hat{\mathbf{k}}$ are the unit vectors in the photon and pion directions, respectively. At leading order in HBCPT, we have $\mathcal{A}_{1}=e g_{A} / \sqrt{2} F_{\pi}, \quad \mathcal{A}_{2}=\mathcal{A}_{1} \omega|\mathbf{k}| / q \cdot k, \quad \mathcal{A}_{3}=-\mathcal{A}_{1} \mathbf{k}^{2} /$ $q \cdot k$, and $\mathcal{A}_{4}=0[26,27]$. As explained in [20] one also requires the nonvanishing subleading order result for $\mathcal{A}_{4}$,

$$
\begin{aligned}
\mathcal{A}_{4}= & \frac{e g_{A}|\mathbf{k}|}{2 \sqrt{2} F_{\pi} m_{N}}\left[\mu_{p}-\left(\frac{\omega}{\omega_{\pi}}\right) \mu_{n}\right] \\
& -\frac{2 e g_{\pi N \Delta} G_{1}|\mathbf{k}|}{9 \sqrt{2} F_{\pi} m_{N}}\left(\frac{\omega}{\omega-\delta}+\frac{\omega}{\omega_{\pi}+\delta}\right),
\end{aligned}
$$

where the $\Delta$ (1232) contribution has been included explicitly. Here $G_{1}$ is the $M 1$ transition moment connecting the nucleon and delta, and $g_{\pi N \Delta}$ is the $\pi-N-\Delta$ coupling [25]. Note that $\mathcal{A}_{1-3}$ is $O\left(p^{0}\right)$ while $\mathcal{A}_{4}$ is $O(p)$.

To $O(p)$ in the chiral expansion, the PV $\gamma p \rightarrow \pi^{+} n$ $T$ matrix can be written as 


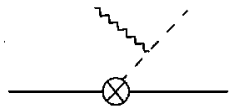

a)

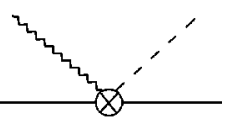

b)

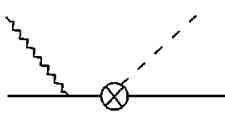

c)

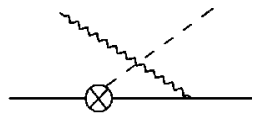

d)
FIG. 1. The relevant Feyman diagrams for PV $\pi^{+}$photoproduction. The circle filled with a cross is the PV vertex.

$$
T^{P V}=N^{\dagger}\left[\mathcal{F}_{1} \hat{\mathbf{k}} \cdot \hat{\epsilon}+i \mathcal{F}_{2} \vec{\sigma} \cdot \hat{\epsilon} \times \hat{\mathbf{q}}+i \mathcal{F}_{3} \vec{\sigma} \cdot \hat{\epsilon} \times \hat{\mathbf{k}}\right] N
$$

We then have the asymmetry

$$
B_{\gamma} \sim\left\{\mathcal{A}_{1} \mathcal{F}_{2}+\frac{\sin ^{2} \theta}{2}\left[\mathcal{A}_{3} \mathcal{F}_{2}-\mathcal{A}_{4} \mathcal{F}_{1}-\mathcal{A}_{2} \mathcal{F}_{3}\right]+\cos \theta \mathcal{A}_{1} \mathcal{F}_{3}\right\},
$$

where $\theta=\cos ^{-1} \hat{\mathbf{q}} \cdot \hat{\mathbf{k}}$. (Note that the nominally leading piece from the interference term $\mathcal{A}_{1-3} \mathcal{F}_{1}$ vanishes if the proton target is unpolarized.)

The leading, nonvanishing contributions to $B_{\gamma}$, which occur at $O\left(p^{0}\right)$, are generated by the $O\left(p^{0}\right)$ terms in $\mathcal{A}_{1-3}$ interfering with the $O\left(p^{0}\right)$ terms in $\mathcal{F}_{2}$, and by the $O(p)$ term in $\mathcal{A}_{4}$ interfering with the $O\left(p^{-1}\right)$ term in $\mathcal{F}_{1}$. The leading order PV contributions to $\mathcal{F}_{1,2}$ arise from the insertion of the PV Yukawa $\pi N N$ vertex of Eq. (13) in Figs. 1(a), 1(c), and 1(d). The results, given in Ref. [20], are

$$
\mathcal{F}_{1}=-\frac{e h_{\pi}^{1}|\mathbf{k}|}{q \cdot k}, \quad \mathcal{F}_{2}=-\frac{e h_{\pi}^{1}}{2 m_{N}}\left[\mu_{p}-\left(\frac{\omega}{\omega_{\pi}}\right) \mu_{n}\right],
$$

where $\mathcal{F}_{1}, \mathcal{F}_{2}$ are $O\left(p^{-1}\right), O\left(p^{0}\right)$, respectively.

Subleading contributions to $B_{\gamma}$ are generated by $O(p)$ and $O\left(p^{2}\right)$ terms in $\mathcal{A}_{1-3}$ and $\mathcal{A}_{4}$, respectively, interfering with the amplitudes in Eq. (19), and by $O(p)$ contributions in $\mathcal{F}_{2,3}$ interfering with the $O\left(p^{0}\right)$ terms in $\mathcal{A}_{1-3}$. The subleading PC contributions have been computed in [27]. We refer to the detailed expressions for these corrections in that work, which we employ in our numerical analysis below. Of greater interest are the $O(p)$ PV amplitudes involving new LEC's. These contributions, which are generated by the $h_{V}$ and $C$ terms in Eq. (13), contribute to both the pole diagrams Figs. 1(c) and 1(d) and the seagull diagram Fig. 1(b). We have

$$
\begin{gathered}
\mathcal{F}_{1}=\mathcal{F}_{3}=0 \\
\mathcal{F}_{2}=\frac{e h_{V}}{2 m_{N}} \frac{\omega_{\pi}}{\sqrt{2} F_{\pi}}\left[\mu_{p}-\frac{\omega}{\omega_{\pi}} \mu_{n}-\frac{\omega}{\omega_{\pi}}\right]+\frac{2 e C}{\Lambda_{\chi}} \frac{\omega}{F_{\pi}} .
\end{gathered}
$$

The contribution from Fig. 1(b) cancels exactly those from Fig. 1(c) and 1(d) where the $\gamma N N$ vertex is minimum coupling. ${ }^{2}$

According to the expression in Eq. (20), the $h_{V}$ and $C$ contributions to $\mathcal{F}_{2}$ carry distinct kinematic dependences, a feature which might suggest using the $\omega$ dependence of $B_{\gamma}$ to separate the two LEC's. Such a program would be misguided, however. As we show below, the kinematic behavior

\footnotetext{
${ }^{2}$ We thank J.-W. Chen and X. Ji for pointing out this cancellation
} to us. generated by the $h_{V}$ and $C$ interactions is identical when a fully relativistic framework is used to compute the PV amplitudes. The result in this case is

$$
\mathcal{F}_{2}=\frac{2 e \bar{C}}{\Lambda_{\chi}} \frac{\omega}{F_{\pi}}
$$

with

$$
\bar{C}=C+\frac{\Lambda_{\chi}}{m_{N}}\left(\frac{\kappa_{p}-\kappa_{n}}{4 \sqrt{2}}\right) h_{V}
$$

Here $\kappa_{i}$ are the anomalous nucleon magnetic moments, as distinguished from the full moments $\mu_{i}$ used to this point. The apparent difference between Eqs. (20) and (21) is an artifact of truncating the $1 / m_{N}$ expansion at this order in HBCPT - to this order of the chiral expansion the photon and pion energies are equal. In what follows, then, we adopt the result in Eq. (21).

In addition to the $O(p)$ contributions from $h_{V}$ and $C, \mathcal{F}_{2}$ receives an $O(p)$ contribution involving $h_{\pi}^{1}$ generated by the $1 / m_{N}$ corrections to the nucleon propagator and $\gamma N N$ vertex in the pole amplitudes. We include these corrections in the asymmetry formulas below. Other possible contributions to the PV amplitudes arise from tree-level graphs containing $\Delta$ intermediate states and from loops. The former require either PV $\gamma N \Delta$ or $\pi N \Delta$ couplings. As discussed in Ref. [17], there exists no PV $\pi N \Delta$ coupling at leading order in $1 / m_{N}$, so that the corresponding amplitudes first appear at $O\left(p^{2}\right)$. Indeed, angular momentum arguments require that the PV $\pi N \Delta$ coupling must be $D$-wave and hence $O\left(p^{2}\right)$. Similarly, the amplitude generated by the PV $\gamma N \Delta$ coupling goes as $\partial_{\lambda} \pi F^{\lambda \mu} \bar{N} \gamma_{\mu} N$, yielding $O\left(p^{2}\right)$ contributions to the $\mathcal{F}_{i}$. Chiral loop contributions to $\mathcal{A}_{1-4}, \mathcal{F}_{1}, \mathcal{F}_{2}, \mathcal{F}_{3}$ appear at $O\left(p^{2}\right)$, $O(p), O\left(p^{2}\right)$ or higher, respectively. In particular, the PV amplitudes receive no contributions from loops containing the leading order PV $\pi N N$ Yukawa coupling and the PC $\gamma \pi \pi$ or PC $\gamma \pi N$ interactions. Such loops require both a $\pi^{+}$ in the intermediate state and emission of a $\pi^{+}$from the intermediate nucleon, and therefore must vanish by charge conservation. The analogous loop containing a $\Delta$ intermediate state and the PV $\pi \Delta \Delta$ coupling is nominally $O(p)$. However, using an explicit calculation, we find that the integral vanishes at this order due to the spacetime structure of the integrand. All remaining loop contributions contain either (i) PV $\pi \pi N N$ or $\gamma \pi \pi N N$ couplings or (ii) the PV $\pi N N$ Yukawa interaction with a $\gamma$-insertion on the intermediate nucleon line. All such diagrams generate sub-sub-leading contributions. Consequently, chiral loops do not contribute to the asymmetry until at least $O\left(p^{2}\right)$ and need not be included in the present analysis.

The resultant photon asymmetry at order $O(p)$ reads then 


$$
\begin{aligned}
B_{\gamma}(\omega, \theta)= & \frac{\sqrt{2} h_{\pi}^{1} F_{\pi}}{g_{A} m_{N} \mathcal{G}}\left\{\left[\left(1-\frac{\omega}{2 m_{N}}\right) \mu_{p}-\left(\frac{\omega}{\omega_{\pi}}\right)\left(1-\frac{|\mathbf{k}|^{2}}{2 m_{N} \omega_{\pi}}\right) \mu_{n}\right]\left(1-\sin ^{2} \theta \frac{\mathbf{k}^{2}}{q \cdot k}\right)+\frac{2}{9} g_{\pi N \Delta} G_{1} \sin ^{2} \theta \frac{\mathbf{k}^{2}}{g_{A} q \cdot k}\right. \\
& \left.\times\left(\frac{\omega}{\omega-\delta}+\frac{\omega}{\omega_{\pi}+\delta}\right)+2\left(\frac{\omega}{\omega_{\pi}}\right) \frac{|\mathbf{k}|}{m_{N}} \mu_{n}\left(\cos \theta-\sin ^{2} \theta \frac{\omega|\mathbf{k}|}{2 q \cdot k}\right)\right\}-\frac{4 \sqrt{2} \bar{C}}{g_{A} \Lambda_{\chi} \mathcal{G}}\left(1-\sin ^{2} \theta \frac{\mathbf{k}^{2}}{2 q \cdot k}\right)+\cdots,
\end{aligned}
$$

where the ellipses indicate the PC $1 / m_{N}$ contributions of $T^{P C}$ in Ref. [27] and

$$
\mathcal{G}=1-\sin ^{2} \theta \frac{\mathbf{k}^{2}}{q \cdot k}\left[1-\frac{(\mathbf{q}-\mathbf{k})^{2}}{2 q \cdot k}\right] .
$$

At threshold, $|\mathbf{k}|=0$, Eq. (23) becomes the low energy theorem for the photon asymmetry given in Eq. (4).

\section{FIELD REDEFINITION AND PHYSICAL OBSERVABLES}

In response to an earlier version of this paper, $\mathrm{CJ}$ observed that one may obtain the subleading PV contributions to $B_{\gamma}$ involving $\bar{C}$ entirely from the diagram (b) in Fig. 1 after a suitable redefinition of the nucleon fields [29]. This simplification arises because the $h_{V}$ terms in Eq. (13) vanish for on-shell nucleons after integration by parts. As discussed in Ref. [30], the effects of interactions which vanish by the equations of motion can always be absorbed into contact interactions via field redefinition. In the present case, the redefinition proposed by $\mathrm{CJ}$ is

$$
\begin{aligned}
& p=\tilde{p}-\frac{i}{\sqrt{2} F_{\pi}} h_{V} \pi^{+} \tilde{n}, \\
& n=\tilde{n}-\frac{i}{\sqrt{2} F_{\pi}} h_{V} \pi^{-} \tilde{p},
\end{aligned}
$$

The resultant PV Lagrangian $\widetilde{\mathcal{L}}^{P V}$ is

$$
\begin{aligned}
\tilde{\mathcal{L}}^{P V}= & -i h_{\pi}^{1} \pi^{+} \overline{\tilde{p}} \tilde{n}+i \frac{h_{A}^{1}+\bar{h}_{A}^{2}}{F_{\pi}^{2}} \overline{\tilde{p}} \gamma^{\mu} \gamma_{5} \tilde{p} \pi^{+} D_{\mu} \pi^{-} \\
& +i \frac{h_{A}^{1}-\bar{h}_{A}^{2}}{F_{\pi}^{2}} \overline{\tilde{n}} \gamma^{\mu} \gamma_{5} \tilde{n} \pi^{+} D_{\mu} \pi^{-} \\
& -i e \frac{\bar{C}}{\Lambda_{\chi} F_{\pi}} \overline{\tilde{p}} \sigma^{\mu \nu} F_{\mu \nu} \tilde{n} \pi^{+}+\text {H.c. }+\cdots,
\end{aligned}
$$

where

$$
\bar{h}_{A}^{(2)}=h_{A}^{(2)}-\frac{g_{A}}{2} h_{V}, \quad \bar{C}=C+\frac{\Lambda_{\chi}}{m_{N}}\left(\frac{\kappa_{p}-\kappa_{n}}{4 \sqrt{2}}\right) h_{V} .
$$

Note that in $\widetilde{\mathcal{L}}^{P V}$, the $h_{V}$ terms have been eliminated, and their effect absorbed into the LEC $\bar{C}$ and $\bar{h}_{A}^{(2)}$ introduced earlier. ${ }^{3}$ In terms of physical observables involving up to two $\pi$ and one $\gamma$, it is not possible to determine $h_{V}$ from $C$. In particular, as noted in Ref. [18], the PV $N N$ potential contains no dependence on $h_{V}$.

The question remains as to whether the $h_{V}^{i}$ constitute distinct LEC's in the context of the full nonlinear Lagrangian of Eq. (9), or whether their effects can be entirely absorbed into other LEC's. In the following, we address this question using the simplest unitarized version of the transformation in Eq. (25). We show that at $3 \pi$ order, it is not possible to eliminate the $h_{V}^{i}$ effects in terms of other LEC's. We give a general proof of this result in the Appendix. In principle, then, one could use an appropriate PV $3 \pi$ process (e.g., the analyzing power for $\pi^{-} \vec{p} \rightarrow \pi^{+} \pi^{-} n$ ) to separate the $h_{V}^{i}$ and $C$. In practice, measurements of multipion processes would be extremely difficult at best.

To illustrate this result, consider the unitary transformation

$$
N=V_{1} \widetilde{N}
$$

to eliminate the leading linear term after expansion of PV vector pieces in Eq. (9). The explicit expression of $V_{1}$ is

$$
\begin{gathered}
V_{1}=e^{-\left(i / F_{\pi}\right) \hat{O}_{1}}=V e^{-\left(i h_{V}^{1} / F_{\pi}\right) \pi^{0}}, \\
V=e^{-\left(i / F_{\pi}\right) \hat{O}},
\end{gathered}
$$

$$
\hat{O}_{1}=\hat{O}+h_{V}^{1} \pi^{0} \hat{1}
$$

$$
\hat{O}=\frac{h_{V}^{0}}{2} \pi^{i} \tau^{i}+\frac{4}{3} h_{V}^{2}\left(\begin{array}{ll}
2 \pi^{0} & \frac{\pi^{+}}{\sqrt{2}} \\
\frac{\pi^{-}}{\sqrt{2}} & -2 \pi^{0}
\end{array}\right) \text {. }
$$

The difference between the field redefinition Eq. (25) and Eq. (28) is twofold. The latter is unitary and also takes into account the PV vector $\pi^{0} N N$ interaction.

It is useful to collect some relevant terms of the redefined Lagrangians containing the nucleon field $\widetilde{N}$. For the strong and electromagnetic part we have

\footnotetext{
${ }^{3}$ Our relative phase between $C$ and $h_{V}$ in $\bar{C}$ differs from Ref. [29].
} 


$$
\begin{aligned}
\widetilde{\mathcal{L}}^{P C}= & \overline{\tilde{N}}\left(i D_{\mu} \gamma^{\mu}-m_{N}\right) \widetilde{N}+\overline{\tilde{N}}\left[V_{1}^{\dagger} i D_{\mu} V_{1}\right] \gamma^{\mu} \widetilde{N}+\overline{\tilde{N}}\left[V^{\dagger} i V_{\mu} V\right] \gamma^{\mu} \widetilde{N}+g_{A} \overline{\tilde{N}}\left[V^{\dagger} A_{\mu} V\right] \gamma^{\mu} \gamma_{5} \widetilde{N} \\
& +\frac{e}{\Lambda_{\chi}} \overline{\tilde{N}}\left[V^{\dagger}\left(c_{s}+c_{v} \tau_{3}\right) \sigma^{\mu \nu} F_{\mu \nu}^{+} V\right] \widetilde{N}+\cdots,
\end{aligned}
$$

where $V_{\mu}$ is the chiral connection.

For the originally weak interaction we have

$$
\begin{aligned}
\widetilde{\mathcal{L}}^{P V}= & h_{V}^{0} \overline{\tilde{N}} V^{\dagger} A_{\mu} V \gamma^{\mu} \widetilde{N}+\frac{1}{2} h_{V}^{1} \tilde{\tilde{N}} \gamma^{\mu} \widetilde{N} \operatorname{Tr}\left(A_{\mu} X_{+}^{3}\right)-\frac{1}{2} h_{A}^{1} \tilde{\tilde{N}} \gamma^{\mu} \gamma_{5} \tilde{N} \operatorname{Tr}\left(A_{\mu} X_{-}^{3}\right)-\frac{1}{2 \sqrt{2}} h_{\pi}^{1} F_{\pi} \tilde{\tilde{N}}\left[V^{\dagger} X_{-}^{3} V\right] \widetilde{N} \\
& +h_{V}^{2} \mathcal{I}^{a b} \tilde{\tilde{N}} V^{\dagger}\left[X_{R}^{a} A_{\mu} X_{R}^{b}+X_{L}^{a} A_{\mu} X_{L}^{b}\right] V \gamma^{\mu} \widetilde{N}-\frac{1}{2} h_{A}^{2} \mathcal{I}^{a b} \tilde{\tilde{N}} V^{\dagger}\left[X_{R}^{a} A_{\mu} X_{R}^{b}-X_{L}^{a} A_{\mu} X_{L}^{b}\right] V \gamma^{\mu} \gamma_{5} \widetilde{N} \\
& +\frac{1}{\Lambda_{\chi}} c_{1} \tilde{\tilde{N}} \sigma^{\mu \nu}\left[F_{\mu \nu}^{+}, X_{-}^{3}\right]_{+} \widetilde{N}+\frac{1}{\Lambda_{\chi}} c_{2} \tilde{\tilde{N}} \sigma^{\mu \nu} F_{\mu \nu}^{-} \tilde{N}+\frac{1}{\Lambda_{\chi}} c_{3} \tilde{\tilde{N}} \sigma^{\mu \nu}\left[F_{\mu \nu}^{-}, X_{+}^{3}\right]_{+} \widetilde{N}
\end{aligned}
$$

Now expand Eqs. (33) and (34) in $1 / F_{\pi}$. The leading term arising from

$$
\overline{\tilde{N}}\left[V_{1}^{\dagger} i D_{\mu} V_{1}\right] \gamma^{\mu} \widetilde{N}
$$

in Eq. (33) entirely cancels the $1 \pi h_{V}^{i}$ terms in Eq. (34), recovering the results of Eqs. (26) and (27). The potential sources of $3 \pi \mathrm{PV}$ interactions include the following.

(1) Expansion of the term in Eq. (33) $\tilde{\tilde{N}}\left[V_{1}^{\dagger} i D_{\mu} V_{1}\right] \gamma^{\mu} \widetilde{N}$ in Eq. (33). The result is $O\left(G_{F}^{3}\right)$.

(2) Expansion of the term in Eq. (33) $\tilde{\tilde{N}}\left[V^{\dagger} i V_{\mu} V\right] \gamma^{\mu} \tilde{N}$, which is linear in $h_{V}^{i}, i=0,2$ only $\left[O\left(G_{F}\right)\right]$.

(3) Expansion of $A_{\mu}, X_{ \pm}^{3}, X_{L, R}^{a}$ operators in Eq. (34) to third order, which is linear in $h_{V}^{i}(i=0,1,2)$ and $h_{A}^{i}, h_{\pi}^{1}(i$ $=1,2)\left[O\left(G_{F}\right)\right]$.

(4) Expansion of $V$ and $V^{\dagger}$ operator in Eq. (34) to second order, which is cubic in $h_{V}^{i}, i=0,2$ only $\left[O\left(G_{F}^{3}\right)\right]$.

(5) Expansion of the $\tilde{\tilde{N}}\left[V^{\dagger} A_{\mu} V\right] \gamma^{\mu} \gamma^{5} \widetilde{N}$ and $\tilde{\tilde{N}}\left[V^{\dagger}\left(c_{s}\right.\right.$ $\left.\left.+c_{v} \tau_{3}\right) \sigma^{\mu \nu} F_{\mu \nu}^{+} V\right] \tilde{N}$ terms in to third order $\left[O\left(G_{F}^{2}\right)\right.$ and $O\left(G_{F}\right)$, respectively].

(6) Expansion of the $c_{i}$ terms in Eq. (34) to third order $\left[O\left(G_{F}\right)\right]$.

Prior to the applying the transformation (28),(29), the only PV $N N \pi \pi \pi$ contact interactions arise from the $h_{V}^{i}$ terms in (3). After field redefinition, one must add up all six contributions. Note that those arising from (5) and (6) and the $h_{\pi}^{1}, h_{A}^{i}$ terms in (3) contain a different Lorentz structure than the $h_{V}^{i}$ terms in (3) and therefore cannot cancel the latter. Similarly, since the $h_{V}^{i} 3 \pi$ terms in (3) arise at $O\left(G_{F}\right)$, they cannot be cancelled by the contributions from (1) and (4). Thus, at $O\left(G_{F}\right)$, the only $3 \pi$ contributions involving $\tilde{N} \gamma_{\mu} \widetilde{N}$ arise from (2) and the $h_{V}^{i}$ terms in (3). Note that (2) contains no terms involving $h_{V}^{1}$. Hence, the $3 \pi$ term proportional to $h_{V}^{1}$ appearing in (3) cannot be removed by the transformation Eq. (28).

For the terms proportional to $h_{V}^{0}$ we obtain from (2)

$$
-\frac{h_{V}^{0}}{2 F_{\pi}^{3}}\left[\pi,\left[\pi, D_{\mu} \pi\right]\right]
$$

where $\pi=\frac{1}{2} \pi^{i} \tau^{i}$, while (3) yields

$$
+\frac{h_{V}^{0}}{6 F_{\pi}^{3}}\left[\pi,\left[\pi, D_{\mu} \pi\right]\right] .
$$

Their sum is

$$
-\frac{h_{V}^{0}}{3 F_{\pi}^{3}}\left[\pi,\left[\pi, D_{\mu} \pi\right]\right] .
$$

The $3 \pi$ PV vector $h_{V}^{0}$ contact term does not vanish after field redefinition. A similar result holds for $h_{V}^{2}$.

As we show in the Appendix, one may remove the $1 \pi h_{V}^{i}$ terms by a more general field redefinition than given by Eqs. (28) and (29). Nevertheless, it is still not possible to remove the $3 \pi$ terms proportional to the $h_{V}^{i}$ (the arguments of the proof are similar to those above, but more tedious in the details). Thus, we conclude that the $h_{V}^{i}$ constitute distinct and, in principle measurable LEC's in the nonlinear chiral theory of Eqs. (5) and (9). While one could compute observables in either formulation of the theory (with or without the field redefinition) and obtain identical results, the structure of Lagrangian is more cumbersome after application of Eq. (28): there appear several new interaction vertices, including small $\left[O\left(G_{F}^{2}\right)\right]$ parity-conserving nonderivative interactions; the chiral transformation properties are less transparent than in the original version of the theory; and the fields $\widetilde{N}$ annihilate nucleon states of mixed parity. Consequently, we retain the original form of $\mathcal{L}^{P V}$ given in Refs. $[4,18]$.

\section{SCALE OF THE LEC'S}

Given that $h_{\pi}^{1}$ and $\bar{C}$ appear in $B_{\gamma}$ with nearly equal weight, it would be useful to have in hand a theoretical expectation for the magnitudes of these LEC's. A simple esti- 
mate can be obtained by applying the "naive dimensional analysis" of Ref. [31]. For strong and EM interactions, effective interactions scale with $F_{\pi}$ and $\Lambda_{\chi}$ as

$$
\left(\Lambda_{\chi} F_{\pi}\right)^{2} \times\left(\frac{\bar{N} N}{\Lambda_{\chi} F_{\pi}^{2}}\right)^{k}\left(\frac{\pi}{F_{\pi}}\right)^{l}\left(\frac{D_{\mu}}{\Lambda_{\chi}}\right)^{m},
$$

where $k, l, m$ are integers and $D_{\mu}$ is the covariant derivative. For weak interactions, the same counting applies, multiplied by an overall scale of

$$
g_{\pi} \sim \frac{G_{F} F_{\pi}^{2}}{2 \sqrt{2}}
$$

Thus, one would expect the strength of the PV $N N \pi$ Yukawa interaction to be given by Eqs. (39) and (40) with $k=1, l$ $=1, n=0$ :

$$
\frac{\Lambda_{\chi}}{F_{\pi}} g_{\pi}=4 \pi g_{\pi}
$$

Since the definition of the Yukawa interaction in Eq. (9) contains no explicit factors of $\Lambda$ or $F_{\pi}$, one expects the natural size of this LEC to be given by Eq. (41). Similarly, the $\bar{C}$ interaction, which involves $k=1, l=1, m=2$, should scale as

$$
\frac{1}{\Lambda_{\chi} F_{\pi}} g_{\pi}
$$

However, since the PV $N N \pi \gamma$ contact interaction in Eq. (34) already contains the explicit factors $1 / \Lambda_{\chi}$ and $1 / F_{\pi}$, the coefficient, $\bar{C}$, should be roughly of size $g_{\pi}$.

It is useful to compare these expectations with results of model calculations as well as with experiment. The benchmark SU(6)/quark model calculation of Ref. [1], updated in Ref. [3], gives a "best" estimate for $h_{\pi}^{\text {eff }}$ of $(7-12)$ $\times g_{\pi}$-roughly commensurate with the expectation of Eq. (41). That analysis, however, allows for the Yukawa coupling to be as small as zero and as large as $(20-30) \times g_{\pi}$, owing to uncertainties associated with various $\mathrm{SU}(6)$ reduced matrix elements and quark model inputs. To date, no estimate of $\bar{C}$ has been performed. A simple estimate can be made, however, by assuming the short-distance PV physics is saturated by $t$-channel vector meson exchange. In the purely mesonic sector, one may understand the magnitudes of the $O\left(p^{4}\right)$ LEC's $L_{i}$ using vector meson saturation. For the baryon sector, the same framework was used to estimate the subleading contributions to the nucleon anapole moment [18]. In the present instance, an illustrative contribution in this context is given in Fig. 2, where the $\bar{C}$-amplitude is generated by the PV $\rho N N$ interaction. For the $\rho \pi \gamma$ vertex we use the Lagrangian

$$
\mathcal{L}_{\rho \pi \gamma}^{P C}=e \frac{g_{\rho \pi \gamma}}{4 m_{\rho}} \epsilon^{\mu \nu \alpha \beta} F_{\mu \nu} G_{\alpha \beta}^{-} \pi^{+}+\cdots
$$

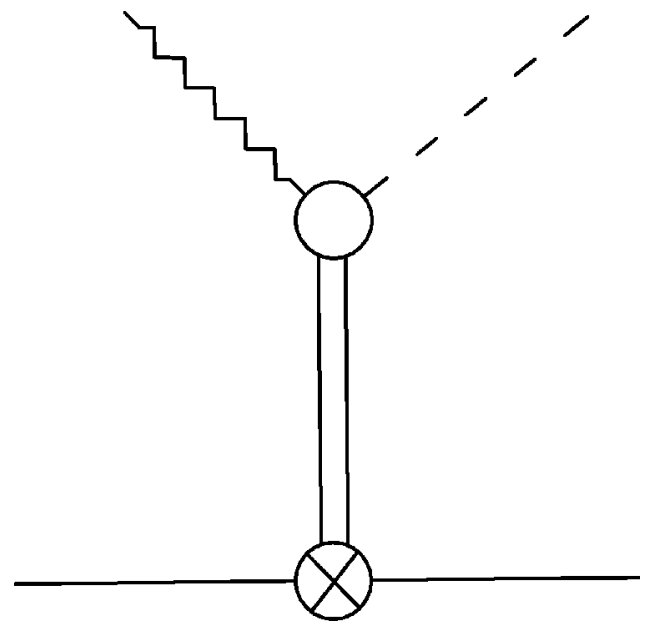

FIG. 2. The $t$-channel $\rho$-meson exchange diagram used to estimate the PV LEC $\bar{C}$.

where $G_{\alpha \beta}=\partial_{\alpha} \rho_{\beta}-\partial_{\beta} \rho_{\alpha}$. From the $\rho$ radiative decay width [28] we have $\left|g_{\rho \pi \gamma}\right|=0.6$, and for the PV $\rho N N$ interaction we follow Ref. [1], writing

$$
\mathcal{L}_{\rho N N}^{P V}=\sqrt{2}\left(h_{\rho}^{0}-\frac{h_{\rho}^{2}}{2 \sqrt{6}}\right)\left[\bar{p} \gamma_{\mu} \gamma_{5} \rho^{+} n+\text { H.c. }+\cdots\right] .
$$

Invoking VMD we have

$$
\bar{C} \sim-\frac{g_{\rho \pi \gamma}}{\sqrt{2}} \frac{\Lambda_{\chi} F_{\pi} m_{\pi}}{m_{\rho}^{3}}\left(h_{\rho}^{0}-\frac{h_{\rho}^{2}}{2 \sqrt{6}}\right) \sim-0.35 g_{\pi},
$$

where we have used the DDH "best values" $h_{\rho}^{0}$ $=-30 g_{\pi}, h_{\rho}^{2}=-25 g_{\pi}$ [1]. Presumably, other heavy mesons contribute with comparable strength. In this simple vector meson saturation picture, then, the size of $\bar{C}$ is consistent with the expectation in Eq. (42). We note that the authors of Ref. [24] adopted similar picture for the short-distance PV physics, treating the $\rho$ and $\omega$ as explicit dynamical degrees of freedom.

As stated at the outset of this work, the quandary for the effective field theory treatment of $B_{\gamma}$ is that the constraints on $h_{\pi}^{1}$ from the $P_{\gamma}\left({ }^{18} \mathrm{~F}\right)$ measurements imply that this coupling is considerably suppressed from its "natural" scale. ${ }^{4}$ While the analysis of Refs. [1,3] can accommodate the ${ }^{18} \mathrm{~F}$ result, one has a more difficult task of explaining this result using effective field theory alone, without reference to the underlying dynamics of strong and weak interactions. Nevertheless, taking the ${ }^{18} \mathrm{~F}$ result at face value implies that in the HBCPT treatment of one- and few-body PV processes nominally sensitive to the PV $\pi N N$ Yukawa coupling, one must also take into consideration subleading PV contribu-

\footnotetext{
${ }^{4}$ The ${ }^{18} \mathrm{~F}$ result is also consistent with the combined results of $\mathrm{PV}$ asymmetry measurements with ${ }^{19} \mathrm{~F}, p+\alpha$, and $p p$ processes (see, e.g., Ref. [11]).
} 
tions as we have done for $B_{\gamma}$. Disentangling the shortdistance physics responsible for these subleading effects then remains an interesting and unsolved problem for both theory and experiment.

Note added in proof. A measurement of $B_{\gamma}$ has been proposed for Jefferson Lab: PR-01-005, R. Suleiman, spokesperson.

\section{ACKNOWLEDGMENTS}

We thank J.-W. Chen, X. Ji, and J.L. Friar for several helpful conversations. This work was supported in part under U.S. Department of Energy Contract Nos. DE-AC0584ER40150 and DE-FG02-00ER41146, the National Science Foundation, and a National Science Foundation Young Investigator Grant.

\section{APPENDIX}

We present here a general proof that the PV $3 \pi$ vector interaction vertex (proportional to the $h_{V}^{i}$ ) cannot be removed by any unitary transformation $U$. To simplify notation, we absorb the factor $1 / F_{\pi}$ into the pion field. From now on it is understood that

$$
\pi=\frac{1}{F_{\pi}} \pi^{i} \frac{\tau^{i}}{2} .
$$

Since the transformation is unitary, we have

$$
\begin{gathered}
\hat{U}=e^{-i \hat{F}}, \\
\hat{F}=\hat{F}^{\dagger} .
\end{gathered}
$$

The operator $\hat{F}$ can be expanded in terms of the number of pions. Since $\hat{F}$ should not carry explicit Lorentz indices, any derivatives should appear in pairs. Because we are discussing $3 \pi$ PV vertex with only one derivative in the present case, the possible derivative terms are irrelevant here. Consequently, we omit them from the following discussion. We also consider explicitly only the $h_{V}^{0,1}$ contributions; the arguments involving $h_{V}^{2}$ are similar, but considerably more tedious.

Expand $\hat{F}$ :

$$
\hat{F}=\hat{O}_{1}+\hat{O}_{2}+\hat{O}_{3}+\cdots,
$$

where $\hat{O}_{n}$ contains products of $n \pi$ fields. The leading term $\hat{O}_{1}$ is needed to remove the $1 \pi \mathrm{PV}$ vector linear term. Its structure is fixed and of $O\left(G_{F}\right)$ as discussed in Sec. IV. The remaining terms $O_{n}, n>1$ could, in principle, be of $O\left(G_{F}^{0}\right)$. In the present case, we need to consider only the terms through $n=3$. The most general forms of $\hat{O}_{2}, \hat{O}_{3}$ read

$$
\begin{aligned}
\hat{O}_{2}= & \left(a_{1} \pi^{+} \pi^{-}+a_{2} \pi^{0} \pi^{0}\right) \hat{1}+\left(a_{3} \pi^{+} \pi^{-}+a_{4} \pi^{0} \pi^{0}\right) \tau_{3} \\
& +a_{5} \pi^{0}\left(\pi^{+} \tau_{+}+\pi^{-} \tau_{-}\right)+i a_{6} \pi^{0}\left(\pi^{+} \tau_{+}-\pi^{-} \tau_{-}\right),
\end{aligned}
$$

$$
\begin{aligned}
\hat{O}_{3}= & \left(b_{1} \pi^{+} \pi^{-}+b_{2} \pi^{0} \pi^{0}\right) \pi^{0} \hat{1}+\left(b_{3} \pi^{+} \pi^{-}+b_{4} \pi^{0} \pi^{0}\right) \pi^{0} \tau_{3} \\
& +\left(b_{5} \pi^{+} \pi^{-}+b_{6} \pi^{0} \pi^{0}\right)\left(\pi^{+} \tau_{+}+\pi^{-} \tau_{-}\right) \\
& +i\left(b_{7} \pi^{+} \pi^{-}+b_{8} \pi^{0} \pi^{0}\right)\left(\pi^{+} \tau_{+}-\pi^{-} \tau_{-}\right),
\end{aligned}
$$

where $a_{1-6}, b_{1-8}$ are real numbers.

Now perform the unitary transformation

$$
N=\hat{U} \tilde{N} .
$$

The possible sources of PV vector $3 \pi$ vertices in the transformed Lagrangians are the same as discussed in Sec. IV [items (1)-(6), but the order in $G_{F}$ is not a priori fixed here]. In addition, we must also expand the $X_{ \pm}^{a}$ along with $A_{\mu}$ in item (3). As was done previously, we may neglect those terms whose Lorentz structure differs from $\bar{N} \gamma_{\mu} \widetilde{N}$. Thus, we consider only the vector terms arising from (1)-(4) (with $V$ $\rightarrow \hat{U}$ ). From (1) we obtain the three $\pi$ contribution

$$
\hat{U}^{\dagger} i D_{\mu} \hat{U}=D_{\mu} \hat{O}_{3}+i\left[\hat{O}_{1}, D_{\mu} \hat{O}_{2}\right]-i\left[D_{\mu} \hat{O}_{1}, \hat{O}_{2}\right]+O\left(\hat{O}_{1}^{3}\right),
$$

where the $\hat{O}_{1}^{3}$ term is $O\left(G_{F}^{3}\right)$ and may be neglected. Since the component of $\hat{O}_{1}$ proportional to $h_{V}^{1}$ is independent of the $\tau^{a}$, it does not contribute to the commutators in Eq. (A8). Hence, we may replace $\hat{O}_{1} \rightarrow \hat{O}$ in the expression above. Since the $\hat{O}_{2,3}$ are may be of $O\left(G_{F}^{0}\right)$, item (1) will generate relevant $3 \pi$ terms under the general unitary transformation.

From item (2) we obtain

$$
\hat{U}^{\dagger} i V_{\mu} \hat{U}=-\left[\hat{O}_{1}, V_{\mu}^{(2)}\right]+\cdots=-\left[\hat{O}, V_{\mu}^{(2)}\right]+\cdots,
$$

where $V_{\mu}^{(2)}$ denotes the $2 \pi$ terms in $V_{\mu}$.

Next, consider the contributions from item (3), including the expansion of the $X_{ \pm}^{3}$. The term proportional to $h_{V}^{1}$ [we neglect the $O\left(G_{F}^{3}\right)$ ] terms is

$$
\frac{1}{2} h_{V}^{1} \operatorname{Tr}\left[A_{\mu} X_{+}^{3}\right]=\frac{2}{3} h_{V}^{1}\left[\pi^{i} \pi_{i} D_{\mu} \pi^{0}-\pi^{0} \pi_{i} D_{\mu} \pi^{i}\right],
$$

which does not contain $\tau_{ \pm}, \tau_{3}$. In order to remove the above term we also need similar terms with $\hat{1}$ structure from Eqs. (A8) and (A9). The commutators never contribute to $\hat{1}$ structure. So the only possibility is the isoscalar piece of $\hat{C}$,

$$
D_{\mu}\left[b_{1} \pi^{+} \pi^{-} \pi^{0}+b_{2} \pi^{0} \pi^{0} \pi^{0}\right],
$$

which is a total derivative of $3 \pi$ fields, and each term is symmetric under field permutations. However, Eq. (A10) does not display such permutation symmetry. In other words, Eqs. (A10) and (A11) cannot completely cancel each other. 
Thus, the $3 \pi h_{V}^{1}$ piece will remain under any unitary transformation.

Now consider the $h_{V}^{0}$ term in item (3). Expansion of the $A_{\mu}$ operator in $\hat{U}^{\dagger} A_{\mu} \hat{U}$ in Eq. (34) leads to

$$
\begin{aligned}
& \sim \frac{1}{6} h_{V}^{0}\left[\pi,\left[\pi, D_{\mu} \pi\right]\right] \\
& =\frac{1}{6} h_{V}^{0}\left\{\frac{\tau_{3}}{2}\left[2 \pi^{+} \pi^{-} D_{\mu} \pi^{0}-\pi^{0} D_{\mu}\left(\pi^{+} \pi^{-}\right)\right]\right. \\
& \quad+\frac{\tau_{+}}{\sqrt{2}}\left[-\pi^{+}\left(\pi^{+} D_{\mu} \pi^{-}-\pi^{-} D_{\mu} \pi^{+}\right)\right. \\
& \left.+\pi^{0}\left(\pi^{0} D_{\mu} \pi^{+}-\pi^{+} D_{\mu} \pi^{0}\right)\right] \\
& +\frac{\tau_{-}}{\sqrt{2}}\left[-\pi^{-}\left(\pi^{-} D_{\mu} \pi^{+}-\pi^{+} D_{\mu} \pi^{-}\right)\right. \\
& \left.\left.+\pi^{0}\left(\pi^{0} D_{\mu} \pi^{-}-\pi^{-} D_{\mu} \pi^{0}\right)\right]\right\}
\end{aligned}
$$

Finally, from item (4) we obtain for the $h_{V}^{0}$ contribution

$$
\begin{aligned}
\sim i h_{V}^{0}\left[\hat{O}_{2}, A_{\mu}\right]+O\left(\hat{O}_{1}^{3}\right)+\cdots= & i h_{V}^{0}\left[\hat{O}_{2}, A_{\mu}\right]+O\left(G_{F}^{3}\right) \\
& +\cdots
\end{aligned}
$$

Now we require the explicit three $\pi$ expressions from Eqs. (A8), (A9), and (A13) [items (1), (2), and (4)] in addition to the expression in Eq. (A12) [item (3)]. These expressions are linear in the $\tau_{i}$ and $\hat{1}$. For clarity, we first focus on the terms involving $\tau_{3}$. From Eq. (A8) we have

$$
\begin{aligned}
\left.\sim D_{\mu} \hat{O}_{3}\right|_{\tau_{3}}+\left.i h_{V}^{0}\left[\pi, D_{\mu} \hat{O}_{2}\right]\right|_{\tau_{3}}-\left.i h_{V}^{0}\left[D_{\mu} \pi, \hat{O}_{2}\right]\right|_{\tau_{3}} \\
=D_{\mu}\left(b_{3} \pi^{+} \pi^{-} \pi^{0}+b_{4} \pi^{0} \pi^{0} \pi^{0}\right) \tau_{3} \\
\quad+i \sqrt{2} a_{5}\left(h_{V}^{0}+\frac{4}{3} h_{V}^{2}\right)\left[\pi^{+} D_{\mu} \pi^{-}-\pi^{-} D_{\mu} \pi^{+}\right] \pi^{0} \tau^{3} \\
\quad+\sqrt{2} a_{6} h_{V}^{0} \pi^{+} \pi^{-} D_{\mu} \pi^{0} \tau_{3},
\end{aligned}
$$

where have used the following identity:

$$
\begin{aligned}
{\left.\left[\pi, D_{\mu} \hat{O}_{2}\right]\right|_{\tau_{3}}=} & \frac{a_{5}}{\sqrt{2}}\left[\pi^{+} D_{\mu} \pi^{-}-\pi^{-} D_{\mu} \pi^{+}\right] \pi^{0} \tau^{3} \\
& -\frac{i a_{6}}{\sqrt{2}}\left[2 \pi^{+} \pi^{-} D_{\mu} \pi^{0}+\pi^{0} D_{\mu}\left(\pi^{+} \pi^{-}\right)\right] \tau_{3} .
\end{aligned}
$$

The contribution from Eq. (A9) [item (2)] is

$$
-\left.\left[\hat{O}, V_{\mu}\right]\right|_{\tau_{3}}=-\frac{1}{4} h_{V}^{0}\left[2 \pi^{+} \pi^{-} D_{\mu} \pi^{0}-\pi^{0} D_{\mu}\left(\pi^{+} \pi^{-}\right)\right] \tau_{3},
$$

while from Eq. (A12) [item (3)] we obtain

$$
+\frac{1}{12} h_{V}^{0}\left[2 \pi^{+} \pi^{-} D_{\mu} \pi^{0}-\pi^{0} D_{\mu}\left(\pi^{+} \pi^{-}\right)\right] \tau_{3} .
$$

Finally, Eq. (A13) [item (4)] gives

$$
\begin{aligned}
\left.i h_{V}^{0}\left[D_{\mu} \pi, \hat{O}_{2}\right]\right|_{\tau_{3}}= & -i h_{V}^{0} \frac{a_{5}}{\sqrt{2}}\left[\pi^{+} D_{\mu} \pi^{-}-\pi^{-} D_{\mu} \pi^{+}\right] \pi^{0} \tau^{3} \\
& +h_{V}^{0} \frac{a_{6}}{\sqrt{2}} \pi^{0} D_{\mu}\left(\pi^{+} \pi^{-}\right) \tau^{3} .
\end{aligned}
$$

The sum of all four possible sources, i.e., Eqs. (A14), (A16), (A17), and (A18), yields

$$
\begin{aligned}
& D_{\mu}\left(b_{3} \pi^{+} \pi^{-} \pi^{0}+b_{4} \pi^{0} \pi^{0} \pi^{0}\right) \tau_{3} \\
& \quad-\frac{1}{6} h_{V}^{0}\left[2 \pi^{+} \pi^{-} D_{\mu} \pi^{0}-\pi^{0} D_{\mu}\left(\pi^{+} \pi^{-}\right)\right] \tau_{3} \\
& +\sqrt{2} a_{6} h_{V}^{0} \pi^{+} \pi^{-} D_{\mu} \pi^{0} \tau_{3} \\
& +\frac{a_{6}}{\sqrt{2}} h_{V}^{0} \pi^{0} D_{\mu}\left(\pi^{+} \pi^{-}\right) \tau_{3} \\
& +i h_{V}^{0} \frac{a_{5}}{\sqrt{2}}\left[\pi^{+} D_{\mu} \pi^{-}-\pi^{-} D_{\mu} \pi^{+}\right] \pi^{0} \tau^{3} .
\end{aligned}
$$

In order for the transformation (A7) to eliminate the $3 \pi$ vector vertex, the sum in Eq. (A19) must vanish. Note the first four lines and the last line of Eq. (A19) are, respectively, symmetric and antisymmetric under the exchange $\pi^{+} \leftrightarrow \pi^{-}$. The symmetric and antisymmetric terms must vanish separately. The solution is

$$
\begin{gathered}
b_{3}=-\frac{2}{3} h_{V}^{0}, \\
b_{4}=0, \\
a_{5}=0, \\
a_{6}=\frac{1}{\sqrt{2}} .
\end{gathered}
$$

Before considering the remaining $h_{V}^{0}$ terms, we observe that the contributions from item (3) involves only expressions involving the pion fields and $\tau_{3}, \tau_{ \pm}$multiplied by real coefficients. The operator, $\hat{O}_{2}$, which contributes via items (1) and (4), only appears in commutators. As a result, the three $\pi$ terms involving $a_{1-5}$ carry factors of $i$ and, thus, cannot cancel the contributions in (3). Consequently, we set $a_{1-5}=0$ in what follows. 
Now we consider the terms linear in $h_{V}^{0}$ and $\tau_{+}$(the argument for $\tau_{-}$is identical). The sum of these contributions is

$$
\begin{aligned}
\left(b_{6}\right. & \left.+i b_{8}\right) D_{\mu}\left(\pi^{0} \pi^{0} \pi^{+}\right)-\sqrt{\frac{2}{6}} h_{V}^{0} \pi^{0}\left(\pi^{0} D_{\mu} \pi^{+}-\pi^{+} D_{\mu} \pi^{0}\right) \\
& -a_{6} h_{V}^{0}\left[\pi^{0} \pi^{0} D_{\mu} \pi^{+}+\pi^{0} \pi^{+} D_{\mu} \pi^{0}\right] \\
& +\sqrt{\frac{2}{6}} h_{V}^{0} \pi^{+}\left(\pi^{+} D_{\mu} \pi^{-}-\pi^{-} D_{\mu} \pi^{+}\right) \\
& +\left(b_{5}+i b_{7}\right) D_{\mu}\left(\pi^{+} \pi^{+} \pi^{-}\right) .
\end{aligned}
$$

Clearly the last two lines (involving only charged $\pi$ fields) can never cancel each other. The solution for the first two lines to vanish is

$$
\begin{gathered}
a_{6}=-\frac{1}{\sqrt{2}}, \\
b_{6}=-\sqrt{\frac{2}{3}} h_{V}^{0}, \\
b_{8}=0 .
\end{gathered}
$$

Note that the requirements on $a_{6}$ in Eqs. (A20) and (A22) are not consistent. Thus, it is not possible with the transformation (A7) to remove the $h_{V}^{0} 3 \pi$ terms from the PV Lagrangian. Moreover, as observed in Ref. [4], Eq. (9) gives the most general PV $\pi N N$ Lagrangian up to one derivative of pion field. There exist no additional PV vector $\pi N N$ contact interaction terms which start off with three pions. Consequently, the $h_{V}^{i}$ cannot be absorbed as part of other LECs at three pion order.
[1] B. Desplanques, J. F. Donoghue, and B. R. Holstein, Ann. Phys. (N.Y.) 124, 449 (1980).

[2] E. G. Adelberger and W. C. Haxton, Annu. Rev. Nucl. Part. Sci. 35, 501 (1985).

[3] G. B. Feldman et al., Phys. Rev. C 43, 863 (1991).

[4] D. B. Kaplan and M. J. Savage, Nucl. Phys. A556, 653 (1993).

[5] W. Haeberli and B. R. Holstein, in Symmetries and Fundamental Interactions in Nuclei, edited by W. C. Haxton and E. M. Henley (World Scientific, Singapore, 1995), p. 17.

[6] W. T. H. van Oers, Int. J. Mod. Phys. E 8, 417 (1999).

[7] S. A. Page et al., Phys. Rev. C 35, 1119 (1987); M. Bini, T. F. Fazzini, G. Poggi, and N. Taccetti, ibid. 38, 1195 (1988).

[8] C. S. Wood et al., Science 275, 1759 (1997); W. C. Haxton, ibid. 275, 1753 (1997); V. V. Flambaum and D. W. Murray, Phys. Rev. C 56, 1641 (1997); W. S. Wilburn and J. D. Bowman, ibid. 57, 3425 (1998).

[9] V. A. Knyazkov et al., Nucl. Phys. A417, 209 (1984); J. F. Cavagnac, B. Vignon, and R. Wilson, Phys. Lett. 67B, 148 (1997); D. M. Markoff, Ph.D. thesis, University of Washington, 1997.

[10] W. C. Haxton, Phys. Rev. Lett. 46, 698 (1981).

[11] W. C. Haxton, C.-P. Liu, and M. J. Ramsey-Musolf, nucl-th/0101018.

[12] C. Bennett, M. M. Lowry, and K. Krien, Bull. Am. Phys. Soc. 25, 486 (1980).

[13] W. M. Snow et al., Nucl. Instrum. Methods Phys. Res. A 440, 729 (2000).
[14] JLab LOI 00-002, W. van Oers and B. Wojtsekhowski, spokesmen.

[15] P. F. Bedaque and M. J. Savage, Phys. Rev. C 62, 018501 (2000).

[16] J. W. Chen, T. D. Cohen, and C. W. Kao, nucl-th/0009031.

[17] Shi-Lin Zhu, S. Puglia, M. J. Ramsey-Musolf, and B. Holstein, Phys. Rev. D 63, 033006 (2001).

[18] Shi-Lin Zhu, S. Puglia, M. J. Ramsey-Musolf, and B. Holstein, Phys. Rev. D 62, 033008 (2000).

[19] R. Hasty et al., Science 290, 2117 (2000).

[20] J.-W. Chen and X. Ji, Phys. Rev. Lett. 86, 4239 (2001).

[21] E. Jenkins and A. V. Manohar, Phys. Lett. B 255, 558 (1991); 259, 353 (1991).

[22] Ulf-G. Meissner, Int. J. Mod. Phys. E 1, 561 (1992).

[23] R. M. Woloshyn, Can. J. Phys. 57, 809 (1979).

[24] S. P. Li, E. M. Henley, and W-Y. P. Hwang, Ann. Phys. (N.Y.) 143, 372 (1982).

[25] T. R. Hemmert, B. R. Holstein, and J. Kambor, J. Phys. G 24, 1831 (1998).

[26] V. Bernard, N. Kaiser, and U.-G. Meissner, Phys. Lett. B 383, 116 (1996).

[27] H. W. Fearing, T. R. Hemmert, R. Lewis, and C. Unkmeir, hep-ph/0005213.

[28] Particle Data Group, C. Caso et al., Eur. Phys. J. C 3, 1 (1998).

[29] J.-W. Chen and X. Ji, hep-ph/0101290; J.-W. Chen (private communication).

[30] H. W. Fearing and S. Scherer, Phys. Rev. C 62, 034003 (2000); C. Arzt, Phys. Lett. B 342, 189 (1995).

[31] A. Manohar and H. Georgi, Nucl. Phys. B234, 189 (1984). 